\title{
Concerned About Separation
}

\author{
Hafedh Mili ${ }^{1}$, Houari Sahraoui ${ }^{2}$, Hakim Lounis ${ }^{1}$, \\ Hamid Mcheick ${ }^{1}$, and Amel Elkharraz ${ }^{1,2}$ \\ ${ }^{1}$ LATECE, Université du Québec à Montréal, Montréal (QC), Canada \\ \{hafedh.mili, hakim.lounis\}@uqam.ca, hamid_mcheick@uqac.ca \\ ${ }^{2}$ GEODES, Université de Montréal, Montréal (QC), Canada \\ \{sahraouh, ekharraz\}@iro.umontreal.ca
}

\begin{abstract}
The separation of concerns, as a conceptual tool, enables us to manage the complexity of the software systems that we develop. There have been a number of approaches aimed at modularizing software around the natural boundaries of the various concerns, including subject-oriented programming, composition filters, aspect-oriented programming, and our own view-oriented programming. The growing body of experiences in using these approaches has identified a number of fundamental issues such as what is a concern, what is an aspect, which concerns are inherently separable, and which aspects are composable. To address these issues, we need to focus on the semantics of separation of concerns, as opposed to the mechanics (and semantics) of aspect-oriented software development methods. We propose a conceptual framework based on a transformational view of software development. Our framework affords us a unified view of the different aspect-oriented development techniques which enables us a simple expression for the separability issue.
\end{abstract}

\section{Introduction}

"Separation of concerns" is a general problem-solving idiom that enables us to break the complexity of a problem into loosely-coupled, easier to solve, subproblems. Underlying this idiom is the hope that, 1) the subproblems are easier to solve, and 2) the solutions to these subproblems can be composed relatively easily to yield a solution to the original problem. The history of programming languages may be seen as a perennial quest for modularisation boundaries that best map (back) to "natural modularisation boundaries" of requirements. Aspect-oriented software development methods are no different. However, most of the research on AOSD has focused on the semantics of aspects and aspect composition, i.e. the solution domain, as opposed to the semantics of concerns and concern separation and composition, i.e. the problem domain. Yet, the early case studies have shown that these conceptually elegant techniques weren't intuitive to use (see [9], [8], [7]). Further, a great number of users of these techniques were caught up in the "how-to" of language constructs, with no regard for the conceptual appropriateness of the AOSD technique for the problem at hand. Further, the various techniques seem to offer orthogonal, but nonetheless useful constructs, with no clear guidelines (which method is appropriate for which problem).

We believe that better understanding of the AOSD techniques will result from a characterization of, 1) the input of software development, and 2) the process of 
software development, to help characterize, if not identify, which concerns are separable, and which development steps are most likely to affect the separation (or separability) of the resulting artifacts. We propose a conceptual framework based on a transformational view of software development. In this context, all the requirements on a software product, be they functional (related to input/output relations) or otherwise (related to how the output is produced), are inputs in these transformations. These requirements fit into general areas, or concerns, which may end up embodied in separate or same artifacts. We distinguish essential separability and inseparability, which characterize requirements, from accidental separability and inseparability, which characterize the realizations of those requirements in development artifacts. Accidental inseparability can be remedied by better language design and user education. Accidental separability should even be discouraged as the conceptual complexity is often increased, and maintenance of the resulting program is often made harder.

\section{Understanding the Separation of Concerns Problem}

Design is a very complicated cognitive task bringing to bear a host of knowledge types and sources and a myriad of problem solving skills [4]. When the artifacts, themselves, are complex, a number of the conceptual and methodological tools fall apart because of scalability problems. Many researchers have shown that complexity is an essential property of design activities in general, due in part to the inevitably incomplete formulation of the problem, and in part to our inability to cope simultaneously with all of the constraints of a given problem (our bounded rationality [16]).

The separation of concerns technique is a general problem solving heuristic that consists of solving a problem by addressing its constraints, first separately, and then combining the partial solutions with the expectation that, 1) they be composable, and 2) the resulting solution is nearly optimal. For this heuristic to yield satisfactory results, the concerns that we are trying to treat separately must be fairly independent, to start with, so that they don't interfere with each other. Further, the problem solving activity itself needs to yield solutions that are composable. In this section, we try to define the separation of concerns problem for the case of software. In this case, the "problem" is a set of requirements, and the "problem solving" process is the software development process. We first start by characterizing the software development process. In section 2.2, we try frame the separation of concerns problem.

\subsection{A Transformational View of Software Development}

Software development is a complex activity involving a variety of skills and a variety of conceptual and formal tools. For the purposes of reasoning about software development-and perhaps automating some of its steps - researchers and practitioners alike have found it useful to view software development as the process of going from specifications of what is to be done (requirements), to precise specifications of how it is to be done. Dasgupta identified two kinds of requirements in any design problem, empirical requirements, which specify externally observable or empirically determinable qualities that are desired of the artifacts, and conceptual requirements, which specify adherence to a particular style [4]. For the case of software, there are two 
kinds of externally observable qualities, functionality - the what - on one hand, and run-time behavior-the how, including performance, and the like. Accordingly, we see three major categories of requirements for software development:

1. Requirements of functionality. These requirements specify an input/output relationship. To satisfy these requirements, we need a function that takes an input/output relationship and returns a function that returns the output for a given input

2. Run-time requirements. These are requirements on run-time behavior such as performance, distribution, the underlying machine (virtual or otherwise), etc.

3. Requirements on the software artifacts. These requirements deal with things such as modularity, reusability, choice of language, etc.

These correspond closely to the categories of architectural qualities identified by [2]. Describing a program using an executable specification language may be seen as performing a first step of the design process, i.e. ensuring functionality. Later steps can worry about run-time behavior and artifact quality. In practice, these three sets of requirements are addressed simultaneously. Further, except in new projects where a complete system is built from the ground up, new functionality often has to integrate into an existing architecture, which embodies a specific point in the design space that addresses a set of run-time and artifact requirements. However, for the purposes of our presentation, we will assume that the three major design dimensions are commutative; two design transformations $T_{1}$ and $T_{2}$ are said to be commutative if given $D_{i}$, the description of the software at step $i$, we have $T_{2} \mathrm{o} T_{1}\left(D_{i}\right)=T_{1} \mathrm{o} T_{2}\left(D_{i}\right)$ (see e.g. [3]). With this mind, let us propose a first-cut description of software development.

Handling functional requirements. Given a relation $\mathrm{R}: \mathrm{A} \times \mathrm{B}$, we need to obtain a function $\mathrm{f}: \mathrm{A} \rightarrow \mathrm{B}$, such that for all $\mathrm{a} \in \mathrm{A}, \mathrm{f}(\mathrm{a}) \in \operatorname{ImageR}(\mathrm{a})$. We say that $\mathrm{f}($.) is an implementation of $\mathrm{R}$. $\mathrm{R}$ describes the relationship that must exist between the input and the output; $f($.$) provides an effective procedure for computing the output, given$ the input. If $\mathrm{R}(.,$.$) is not a function (i.e. some elements of \mathrm{A}$ have more than one image), then $\mathrm{f}($.) picks one element. Automatic programming consists, to a great extent, of automating the "operationalization of requirements". This transformation may be described by a relation OR: $\{\mathrm{R}\} \times\{\mathrm{f}()$.$\} from the set of relations to the set of func-$ tions. Let $\mathrm{R}$ be the set of relations and $\mathrm{F}$ the set of functions. OR is thus a subset of $\mathrm{R} \times \mathrm{F}$. This relation may be known intensionally, or extensionally (through exemplar pairs). Automating this step consists of finding a function $\mathrm{g}: \mathrm{R} \rightarrow \mathrm{F}$ such that given a relation $R \in R, g(R(.,))=.f($.$) where (R, f().) \in O R$. We say that $g$ is an implementation of OR.

Handling run-time requirements. These include performance requirements and execution model. These requirements are handled differently from functional ones. Whereas the operationalization of requirements associates a requirement with any function that implements the requirement, here we are picky about the properties of such functions. For example, such functions have to be efficiently computed. Instead of the relation $O R$ shown below, we now have a subrelation EOR (Efficient Operationalization of Requirements) such that $E O R \subseteq \mathrm{OR}$, where Domain $(E O R)=$ Domain $(O R)$, but $\operatorname{Image}_{\mathrm{EOR}}(\mathrm{R}) \subseteq \operatorname{Image}_{\mathrm{OR}}(\mathrm{R})$. In other words, out of all the functions that implement $\mathrm{R}$, we pick the ones that are efficient. 
Issues related to the execution model include things such as distribution, synchronization, and security. This does not change the function that is computed but changes things about where the different pieces are executed and how. We can represent the execution of function $\mathrm{f}()$ as follows: $\mathrm{EX}: \underline{\mathrm{F}} \times \mathrm{I} \times \mathrm{M} \rightarrow \mathrm{O} \times \mathrm{M}$. EX takes three arguments: a) the function to be computed, b) its input(s), and c) the initial state of the machine. EX produces a pair of outputs: the result of applying the function to its in-

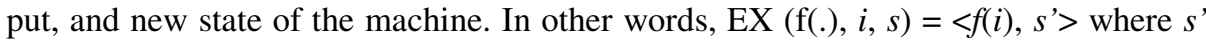
is the state of the machine after it has finished execution of function $f($.) on input $i$. The state changes consist of the side effects of the execution and may involve things such as establishing or terminating connections, modifying the state of data on permanent storage, logging, collecting statistics, etc..

Generally speaking, EX is a composition of several functions. For example, with a virtual machine architecture, we have the hardware machine executing the virtual machine, and the virtual machine executing the actual program. The execution of the virtual machine itself could be written as VM (f(.), $i, s)=\left\langle f(i), s^{\prime}\right\rangle$. The hardware machine, in turn, is executing the function $V M$ on its inputs, and changes state. The input of the $V M$ consists of the triple $\langle f(), i,. \mathrm{~s}>$, where $f($.$) is the function to be exe-$ cuted-written in "virtual machine language"- $i$ is the input of $f($.$) , and s$ the state of VM. Let the hardware machine be represented by the function HM, we have HM (VM(.,...), $(f(), i, s),. h s)=\left\langle<f(i), s^{\prime}>, h s^{\prime}>\right.$. And so forth. The virtual machine itself consists of a set of layered (composed) services or parallel services. An example of layered services is $\mathrm{VM}(\mathrm{f}(), i)=.\mathrm{VM}_{1} \mathrm{O} \mathrm{VM}_{2}(\mathrm{f}(), i, s$.$) . An example of parallel services$ is represented as $<\mathrm{VM}_{1} ; \mathrm{VM}_{2}>(\mathrm{f}(), i, s$.$) where \mathrm{VM}_{1}$ and $\mathrm{VM}_{2}$ are two services that are performed in parallel but such that the end result is the pair $\left\langle f(i), s^{\prime}\right\rangle$. It may be that one service computes the result $\left(\mathrm{VM}_{1}(\mathrm{f}(), i, s)=.f(i)\right)$ while the other changes the state of the machine $\left(\mathrm{VM}_{2}(\mathrm{f}(), i, s)=.s^{\prime}\right)$. We could also have situations where $\mathrm{VM}_{1}$ and $\mathrm{VM}_{2}$ modify different parts of the state of the executing machine. The output itself may be computed by one or two of the virtual machines.

Handling requirements on the artifacts. This involves taking into account the packaging of the function $f($.) based on a number of criteria, including a reasonable division of labor, reusability, cohesion and coupling of the resulting modules, etc. It also includes things such as the choice of a programming language, programming style, etc. Note that requirements on artifacts may lead us to implement more than the initial requirements. For example, reusability considerations may compel us to implement more generic classes to accommodate the needs of other applications within the same domain. It may also compel us to break down functions differently to identify common parts, without necessarily implementing more functionality than required. Let us take a problem $\mathrm{R}($.$) , and its realization, some function f($.$) . Idem for a$ problem R'() with realization $f^{\prime}()$. If we can write $f=f_{\text {post }}$ o $g$ o $f_{\text {pre }}$ and $f^{\prime}=f_{\text {post }}^{\prime}$ o $g$ o $f_{\text {pre }}$, then we reduce the amount of new code to be developed.

\subsection{Framing the Separation of Concerns Problem}

For the purposes of our discussion, we define a concern as a set of related requirements. Elements of the set may be defined extensively (enumerated) or intensively, by referring to a domain (e.g. security). Simply put, requirements are sets of properties 
that must be satisfied by the solution. If we use predicate logic to express requirements, a number of intuitions that we have about requirements have a simple expression in logic [21]. The basic premise of separation of concerns approaches to software development is that requirements have nice properties, and to the extent that we can associate artifacts with concerns, we would like the artifacts to have similar properties! Precisely, the "separation of concerns" methods rely on the existence of a development homomorphism such as the one illustrated in Figure 1. Assume that requirements are represented by predicates, and let $A_{P}=O R(P()$.$) be the artifact that corre-$ sponds to predicate $P($.). Development (represented by the thick arrow) is a homomorphism if there exists an operator $\oplus$ defined on artifacts such that $O R(P(.) \Lambda Q()$. $\equiv O R(P().) \oplus O R(Q()$.$) .$

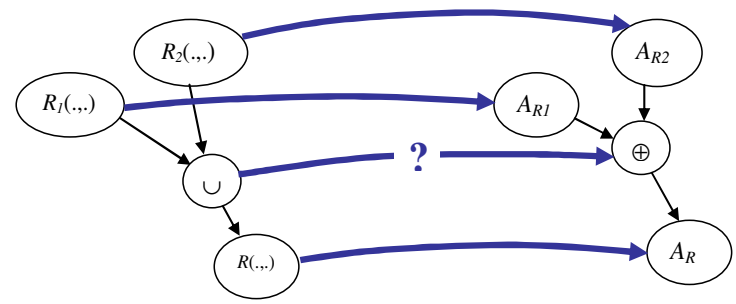

Fig. 1. Development is a homomorphism from requirements to artifacts

We have some intuitions about cases where this homomorphism between requirements and artifacts holds. For example, given two requirements defined by relations $R_{l}: A \rightarrow B$, and $R_{2}: B \rightarrow C$, we know of several operators $\oplus$ such that $O R\left(R_{2} \mathrm{O} R_{l}\right)$ $\equiv O R\left(R_{l}\right) \oplus O R\left(R_{2}\right)$. For example, if the implementation adopts the call-and-return style, the operator $\oplus$ consists of the call relationship between procedures. If the publish-and-subscribe style is used, the operator $\oplus$ consists of registering $O R\left(R_{l}\right)$ as a publisher of some message, and $O R\left(R_{2}\right)$ as a subscriber to that message. Etc.

The advantages of this homomorphism include reusability, configurability, and separate maintenance. A number of object-oriented programming constructs and design idioms may be seen in this light. The new generation of separation of concerns techniques may be seen as defining new modularization boundaries for requirements, that are different from the ones afforded by regular object-oriented programming, and that are realizable in artifacts that are composable according to some composition operator. For example, OORAM uses role models [18] as new behavioral modules, and role synthesis to compose role models. Subject-oriented programming defined subjects [6] as new modular structures, and subject composition, as a composition mechanism [14]. Aspect oriented programming defines aspects as new module boundaries, and aspect-weaving as a way of composing aspects with regular classes [10]. Our own view-oriented programming uses viewpoints as a way of representing domain-independent business processes, and view instantiation and attachment as a way of adding that behavior to objects [12],[13]. All of these techniques may be collectively referred to as aspect oriented development techniques, where composition filters, subjects, aspects à la Kiczales et al., and our views may all be referred to as 
aspects. Thus, we can talk about functional aspects which are associated with functional concerns or architectural aspects which are associated with architectural concerns.

Notwithstanding the case of OORAM, where the emphasis is on requirements level separation (role models) and composition, much of the so-called aspect-oriented development techniques have focused on the mechanics of artifact composition, sometimes losing sight of, 1) the requirements that these artifacts are supposed to embody, and 2) whether that composition (or separation) makes sense, from a requirements point of view. Further, even in those cases where AO techniques seemed appropriate, there were sometimes better non-aspect oriented solutions (see e.g. [15]).

If we view requirements as predicates on the solution, then requirements are clearly composable using logical composition $(\wedge)$-whether the resulting conjunction has solutions or not [21]. However, for the homomorphism of Figure 1 to hold, (1) the requirements that we need to compose have to be independent, and (2) the development transformations have to preserve such independence so that the resulting artifacts (aspects) may be combined.

\section{All Concerns/Aspects Are Functional}

We identified in section 2.1 three distinct kinds of requirements, requirements of functionality, run-time requirements, and requirements on the software artifacts themselves. Before we talk about the conditions under which different requirements (or concerns) may be separable, and whether we should try to untangle or compose their associated artifacts, we look for a common framework that would enable us to look at all three kinds of requirements, and that would enable us to take a simpler view of the separability and composability issue. We start our discussion by first characterizing the ways in which requirements in each category are handled (individually). We will argue that run-time requirements can be represented as functional requirements on the virtual machine; requirements on artifacts are more difficult to formalize.

\subsection{Handling Run-Time Requirements}

We consider run-time requirements to be functional requirements on an imaginary virtual machine that will execute the program in the context of the real machine. The virtual machine will add a number of services including distribution, persistence, security, and others. Persistence services may be seen as providing the program with an execution environment (a virtual machine) that persists automatically the objects that the program manipulates. Most object-oriented databases operate this way (Versant, ObjectStore): developers write programs that manipulate persistent objects in a seamless fashion. It is as if databases come with their own run-time object model, built on top of the host language object model. We later see how this is actually implemented-interestingly, a limited form of aspect-oriented programming.

Distribution is similar to persistence in principle. Lest we oversimplify, distribution may be seen as providing a virtual machine whose run-time representation of objects accommodates remote objects, with what that implies in terms of referencing and in 
terms of method invocation. Consider the following CORBA or RMI-like code sequence:

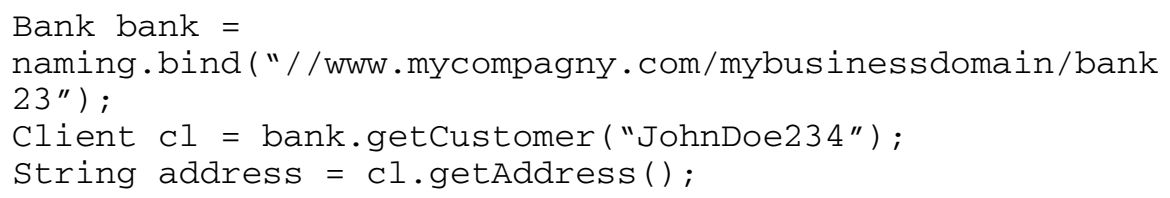

Notwithstanding the first line, which suggests the use of a naming service, the subsequent lines are indifferent from the location of the objects. We could imagine the same program being run in local mode, where the default Java virtual machine runtime representation of objects is used, and "a distributed Java virtual machine" that uses a level of indirection for run-time object representation to access remote objects, and that invokes an ORB to execute methods. Existing implementations of distribution use a slightly different implementation but the idea is the same. In fact, some researchers have even attempted to distribute regular $\mathrm{OO}$ applications using AspectJTM [17].

The way distribution and persistence have been commonly implemented present some commonality. Transparency to the developer dictates a virtual machine metaphor. However, both techniques instrument user code with service-specific code that invokes those services (persistence or remote access). With Java-style persistence (e.g. ObjectStore), the code that is injected is added directly to the compiled Java bytecodes. With distribution, the IDL compiler injects, along with user code, code that is meant to be executed by the distribution virtual machine.

The same can be said about some aspects of security. Both authentication and encryption can be easily (and naturally) implemented at the virtual machine level: one involves encrypting exchanged data (through method calls), and the other authenticates the caller. In fact, Java's own security model is supported and enforced by the virtual machine, which can be thought of as submitting method execution requests to a security manager. J2EE's security model is enforced by the containers-a higher level yet virtual machine.

One reason why virtual machine-like implementations of these services are not common-with the exception of security, for which we want no loopholes-is performance. The other is selectivity: because these services involve an overhead, if we embed it in the virtual machine, then all objects will use it, whether they need it or not. With this code injection mechanism, the code will only be injected in those objects/classes that need it.

As mentioned above, common implementations of persistence use a variant of aspect oriented programming: persistence code is added into designated class files (typically specified in configuration files) so that object creation, accessing, and modification access the database client. The same is true for distribution, where client-side stubs (proxies) go through the ORB to get the data they need. Viewing run-time requirements as functional requirements on the virtual machine helps us understand which services are separable and/or composable, and also helps us understand which solutions are feasible under which situations, and understand some of the anomalies that arise from composing virtual machine-level services. 


\subsection{Handling Requirements on the Artifacts}

Requirements on the artifacts deal with development-time "abilities", with no regard for functionality or performance. Such requirements include understandability, reusability, maintainability, etc. Let $R($.$) be a functional requirement, and f($.$) be an opera-$ tionalization of $R($.$) , i.e. f(.) \in O R(R()$.$) . The various "abilities" on the artifacts can$ typically be written as constraints on various metrics on the artifacts, such as:

- $M_{i}(f())=.\operatorname{MIN}_{\mathrm{g}_{\in} O R(R(.))}\left(M_{i}(g()\right.$.$) (relative constraint) or$

- $M_{i}(f().) \leq \alpha$, for some constant $\alpha$ (absolute constraint)

These meta-level constraints determine the packaging of the functionality.

Separation of concerns is a requirement on software artifacts that is being addressed with AOSD techniques. Thus, our discussion of how development affects separation of concerns will be limited to the development activities related to accommodating functional requirements and those related to handling run-time requirements.

\subsection{Concerns Are Functional While Aspects May Not Be}

Notwithstanding requirements on the artifacts themselves, we have functional requirements and run-time requirements. Run-time requirements are either measurable quality constraints (e.g. performance, space usage), or architectural services. We have shown in section 3.1 that the latter may be thought of as functional requirements on the virtual machine that executes the program. If we take this view, we could view both SOP and AOP, say, as being both concerned with the composition of functional concerns (or the corresponding aspects), with the difference that:

1. SOP (and our own method, VOP) manipulates functional concerns and aspects of the user program directly

2. AOP translates functional concerns on the virtual machine that executes a program $P$, into non-functional aspects to be woven into program $P$.

In fact, a number of researchers have recognized that the kind of concerns that AOP handles well are best (most simply) expressed at the meta-level, and a number of successors to Kiczlaes's AOP use a meta-level architecture to add functionality to the way these machines execute programs-mostly for intercepting message sends to perform processing before or after. In fact, Kiczales himself has said on many occasions that he developed AspectJ ${ }^{\mathrm{TM}}$ as a more constrained/safer version of the MOP to enable "average developers" to add pervasive behavior without compromising the integrity of the VM. Filman \& Friedman consider quantification and obliviousness as essential features of AOP [5]. Both properties can be naturally expressed at the virtual machine level. Steimann that there are no aspects à la AspectJTM for domain models [19]: aspects are solution (read: software) artifacts, and should have no place in domain models or in object-oriented analysis.

If we accept that aspects à la AspectJTM are functional aspects on the virtual machine, we can immediately see that functional concerns and run-time concerns are orthogonal, and we can address them separately, at least up to the analysis step. We can also see that we shouldn't even try to combine functional aspects of the pro$\operatorname{gram}(\mathrm{s})$ that we are developing with functional aspects of the machines that execute 
them! At least not conceptually. And yet, that is what AspectJTM's weavers were explicitly created for!

Composition filters are based on the message passing (and interception) metaphor, but the filters can be either functional, in which case we deal with the normal functional composition, or architectural, in which case, they too, could be handled at the virtual machine level. Thus, for the purposes of understanding the separability/composability of requirements, and the corresponding composability of the associated software artifacts, we need only to focus on the functional separately or composability of (functional) requirements.

\section{Characterizing the Separability of Requirements}

In this section, we attempt the overly ambitious goal of answering two dual questions:

1. Given two requirements, under what conditions can they be "developed" separately, and can their realizations (aspects) be composed at will. The answer to this question will help determine the domain or operating range of the development homomorphism we illustrated in Figure 1. We refer to this problem as the composability of requirement realizations.

2. Given a realization that addresses several concerns, under what conditions can that realization be untangled into separable aspects, each of which addressing a subset of concerns. The answer to this question may help us assess which systems may be re-engineered in such a way that different concerns are addressed in separate-and readily reusable - aspects. We refer to this problem as the separability of requirement realizations.

In addition to its practical importance, an answer to the second question will also help us understand why case studies have not been as convincing as the textbook cases that the original method authors have presented in support of their techniques.

Section 4.1 looks at the composability of requirement realizations problem for the case of functional requirements. We examine the problem from a purely mathematical point of view, reducing the separability of two requirements, seen as (input,output) relations, to conditions on their domains and ranges. This will enable us to address composability issues between runtime requirements or between functional requirements, but not between a functional requirement and a run-time requirement. Section 4.2 tries to answer the separability of requirement realizations for functional requirements by looking at the problem of decomposing a function into separate subfunctions. We look at a range of decomposition/recomposition operators with different semantics preserving properties.

\subsection{Composable Requirements}

Given a development transformation $T$, we consider two requirements $R_{l}$ and $R_{2}$ to be $T$-composable if:

1. we can associate separate realizations to them $\left(T\left(R_{l}\right)\right.$ and $\left.T\left(R_{2}\right)\right)$, and

2. there exists a composition operator $\otimes$ on their realizations that satisfies them both, i.e. $T\left(R_{1} \Lambda R_{2}\right)=T\left(R_{1}\right) \otimes T\left(R_{2}\right)$ 
We showed in section 2.1 that functional requirements are transformed using an operationalization operator-OR, turning an input-output relation into a function that produces the output given the input. Having argued in section 3.1.1 that run-time requirements are nothing but functional requirements on the virtual machine, we look at the problem of composing two functional requirements through the operationalization operator.

We would like the operationalization of functional requirements to be additive at least in those cases where the two requirements have disjoint domains. Consider two relations R and R' such that $\operatorname{Domain}(\mathrm{R}) \cap \operatorname{Domain}\left(\mathrm{R}^{\prime}\right)=\Phi$.The simplest way of implementing $\mathrm{R} \cup \mathrm{R}^{\prime}$ is by taking $f(.) \oplus f^{\prime}($.$) , where f(.) \oplus f^{\prime}()=.\mathrm{g}(\mathrm{x})$ such that:

$$
\begin{aligned}
\mathrm{g}(\mathrm{x}) & =f(\mathrm{x}), \text { if } x \in \operatorname{Domain}(\mathrm{R}) \\
& =f^{\prime}(\mathrm{x}), \text { if } x \in \operatorname{Domain}\left(\mathrm{R}^{\prime}\right)
\end{aligned}
$$

In other words, the simplest $O R($.$) would behave as O R\left(\mathrm{R} \cup \mathrm{R}^{\prime}\right)=f(.) \oplus f^{\prime}($.

Note that if we take into account reuse, then we may be able to write $f=f_{\text {post }}$ o $g$ o $f_{\text {pre }}$ and $f^{\prime}=f^{\prime}$ post o $g$ o $f_{\text {pre }}^{\prime}$. We do have Domain $\left(f^{\prime}{ }_{\text {pre }}\right)=\operatorname{Domain}\left(f^{\prime}\right)$ and $\operatorname{Domain}\left(f_{\text {pre }}\right)$ $=\operatorname{Domain}(f)$, and thus Domain $\left(f_{\text {pre }}\right) \cap \operatorname{Domain}\left(f_{\text {pre }}^{\prime}\right)=\Phi$, but we don't know whether $\operatorname{Domain}\left(f_{\text {post }}\right)$ and $\operatorname{Domain}\left(f_{\text {post }}^{\prime}\right)$ are disjoint, and we can't write $O R(\mathrm{R} \cup \mathrm{R}$ ') (or $\left.f(.) \oplus f^{\prime}().\right)$ as $\left[f_{\text {post }}(.) \oplus f^{\prime}{ }_{\text {post }}().\right]$ o $g$ o $\left[f_{\text {pre }}(.) \oplus f^{\prime}\right.$ pre $\left.().\right]$.

If the relations have intersecting domains, we can define them as follows: $R=R_{1} \cup$ $\mathrm{R}_{2}$ and $\mathrm{R}^{\prime}=\mathrm{R}^{\prime}{ }_{1} \cup \mathrm{R}^{\prime}{ }_{2}$ such that: $\operatorname{Domain}\left(\mathrm{R}_{1}\right)=\operatorname{Domain}(\mathrm{R})$ - Domain $\left(\mathrm{R}^{\prime}\right)$, Do$\operatorname{main}\left(\mathrm{R}_{1}{ }_{1}\right)=\operatorname{Domain}\left(\mathrm{R}^{\prime}\right)-\operatorname{Domain}(\mathrm{R})$, and $\operatorname{Domain}\left(\mathrm{R}_{2}\right)=\operatorname{Domain}\left(\mathrm{R}_{2}\right)=\operatorname{Domain}(\mathrm{R})$ $\cap \operatorname{Domain}\left(R^{\prime}\right)$. In this case, the relation to implement is $R_{1} \cup R^{\prime}{ }_{1} \cup\left(R_{2} \cup R^{\prime}{ }_{2}\right)$, where $\mathrm{R}_{1}, \mathrm{R}^{\prime}{ }_{1}$, and $\mathrm{R}_{2} \cup \mathrm{R}^{\prime}{ }_{2}$ have mutually disjoint domains. Thus, we have $O R\left(\mathrm{R}_{1} \cup \mathrm{R}^{\prime} \cup\right.$ $\left.\left(\mathrm{R}_{2} \cup \mathrm{R}_{2}^{\prime}\right)\right)=O R\left(\mathrm{R}_{1}\right) \oplus O R\left(\mathrm{R}_{2}\right) \oplus O R\left(\mathrm{R}_{2} \cup \mathrm{R}_{2}^{\prime}\right)$.

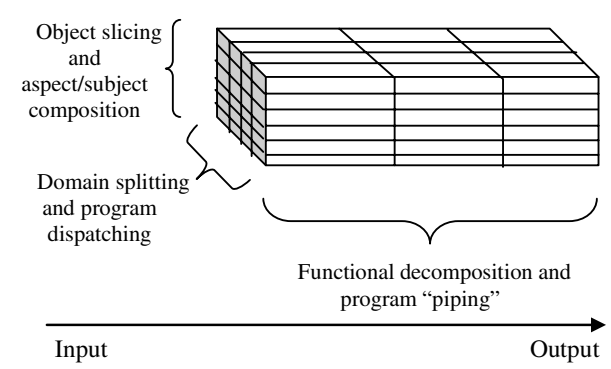

Fig. 2. Comparing three decomposition paradigms

This relationship is trivially satisfied in case $R_{2}=R^{\prime}{ }_{2}$. This is the ideal case in the sense that both requirements agree on what the output should be for the same inputs. In that case, the two requirements $\left(R_{1}\right.$ and $\left.R_{2}\right)$ may be seen as two restrictions of the same relationship defined on the domain $\operatorname{Domain}\left(R_{1}\right) \cup \operatorname{Domain}\left(R^{\prime}{ }_{1}\right)$. If the two relationships disagree on the output, then we have a problem. We see two levels of disagreement. The first level of disagreement is illustrated in the following example. Consider the two relations $R_{1}=\left\{(x, y) \mid 0<x<100\right.$, and $\left.x^{2}=y\right\}$ and $R_{2}=\{(x, y) \mid 50<$ 
$x<150$, and $x^{2}=y$ \}. The intersection of the two domains consists of the interval [50..100]. If both the realizations of $R 1$ and $R 2$ use the positive square root of $x$-or both use the negative square root-then we are fine. If they use different square roots, then we have a problem. This incompatibility is due to an inconsistent choice of realizations, and is a common and acceptable course of action. Intuitively, what we need in this case to make sure that we use consistent realizations. This is not unlike the problem of choosing consistent specializations when we instantiate a framework, i.e. the kind of situations for which things such as the factory pattern is applicable.

The second level of disagreement is the case where the requirements themselves disagree, i.e. $\exists x \in \operatorname{Domain}\left(\mathrm{R}_{1}\right) \cap \operatorname{Domain}\left(\mathrm{R}_{2}\right)$ s.t. $\mathrm{R}_{1}(x) \neq \mathrm{R}_{2}(x)$

In our view, this is not a case for separation of concerns methods to handle: the requirements disagree, so there is no point in trying to compose the artifacts.

\subsection{Separable Requirements}

Given a development transformation $T$, we consider a requirement $R$ (an element of the domain of $T$ ) to be $T$-separable if there exist, 1) two requirements $R_{l}$ and $R_{2}, 2$ ) a composition operator $\bullet$ defined on the domain of $T$ - the requirements—and, 3) a composition operator $\otimes$ on the image of $T$-the artifacts—such that:

1. $\mathrm{R}=\mathrm{R}_{1} \bullet \mathrm{R}_{2}$

2. $\mathrm{T}(\mathrm{R})=\mathrm{T}\left(\mathrm{R}_{1}\right) \otimes \mathrm{T}\left(\mathrm{R}_{2}\right)$

This is the good old divide-and-conquer analytical development paradigm. With structured analysis and design (and programming), the operator is functional composition, in the mathematical sense, and $\otimes$ is "piping", in the programming sense (the output of a program or procedure is used as an input to the other). Functional decomposition is not only useful for reducing complexity, it is also useful for reuse.

Another valuable pair of operators corresponds to the combination of domain splitting and dispatching. Consider the requirement $R$ where domain $(R)=D=D_{1} \oplus D_{2}$. the symbol $\oplus$ referring to disjoint union (partition). Let $T$ be the operationalization of requirements $(O R()$.$) , and R_{l}=R \mid D_{1}$, and $R_{2}=R \mid D_{2}$. Then:

$$
O R(R(.))=\left\{\begin{array}{l}
\text { if } x \in D_{1} \text { call } O R\left(R_{1}\right) \\
\text { if } x \in D_{2} \text { call } O R\left(R_{2}\right)
\end{array}\right.
$$

We are all familiar with these two techniques, and have used them-and should continue to do so- to good measure. Aspect-oriented development techniques advocate other pairs of decompose/recompose or split/join operators which are specific to the object-oriented context. These new pairs of operators operate simultaneously on functions and data, along the lines of object or class hierarchy slicing (see e.g. [20]). In this case, instead of considering the input domain $(D)$ as consisting of simple value, we consider it as a tuple (of state variables), and functions (object methods) may operate on various "sub-tuples".

Figure 2 illustrates the three decomposition paradigms. For each paradigm, we mention the decomposition technique used on requirements, and the corresponding composition technique used on the corresponding artifacts. Now, we look more 
closely at the problem of sliceability of requirements. We start with a strict definition of sliceability which supports unrestricted (commutative) recomposition of the artifacts. We then propose a weaker form of sliceability which requires an ordered (noncommutative) recomposition.

Sliceability. Let $\mathrm{R} \subseteq \mathrm{A} \times \mathrm{B}$, let $\mathrm{f}()=.\mathrm{OR}(\mathrm{R})$, and assume that $\mathrm{A}=\mathrm{S} 1 \times \mathrm{S} 2 \times \ldots \times \mathrm{Si}$ $\times \mathrm{Si}+1 \times \ldots \times \mathrm{Sn} \times \mathrm{I}$ and $\mathrm{B}=\mathrm{S} 1 \times \mathrm{S} 2 \times \ldots \times \mathrm{Si} \times \mathrm{Si}+1 \times \ldots \times \mathrm{Sn} \times \mathrm{O}$. We say that $\mathrm{R}($ or $\mathrm{f}())$ is sliceable if there exist two functions $\mathrm{f} 1(\mathrm{x} 1, \ldots, \mathrm{xi}, \mathrm{i})$ et $\mathrm{f} 2(\mathrm{xi}+1, \ldots, \mathrm{xn}, \mathrm{i})$ such that $f(x 1, \ldots, x i, x i+1, \ldots, x n, i)=f 1(x 1, \ldots, x i, i) \bullet f 2(x i+1, \ldots, x n, i)$. In other words, the function $\mathrm{f}()$ can be computed as the concatenation of two functions.

The idea of sliceability is related to the idea that a relation may be written as a subset of the product of two relations. For example, let $R_{1}$ and $R_{2}$ be two binary relations. We can define the relation $R_{1} \times R_{2}$ as follows: $\left\langle x_{1}, x_{2}, y_{1}, y_{2}\right\rangle \in R_{1} \times R_{2}$ if and only if $<x_{1}, y_{1}>\in R_{1}$ and $<, x_{2}, y_{2}>\in R_{2}$.

Intuitively, the sliceability corresponds to the case where we have two functions that take the same input and that use and modify different parts of an object, i.e. they correspond to two disjoint slices of the same data (or object). Sliceable functions can be put together, with no problem. Notice that we require that both functions take the input (which may be either a real input or a method selector), and that the output is produced between them. In the context of an object-oriented program, if we have a method that returns void but modifies the state of the object, then each subfunction will have modified its slice. If the function returns a value, then we might be able to find a subset of state variables based on which the output is computed, and the slice may be made along that. Note, however, that not all relations/functions are sliceable. A function that averages the state variables will not be sliceable.

Subject-oriented programming (and hyperspaces) works best with this ideal case in mind. Problematic cases occur when the sliceability hypothesis fails. Interestingly, the broken delegation problem can be understood in terms of sliceability of functions. Broken delegation happens when a function that occurs on one side (i.e. in a single object fragment) calls a separable function that occurs on several object fragments (see e.g. [1]): the result is no longer separable.

Effective sliceability. Let $\mathrm{R} \subseteq \mathrm{A} \times \mathrm{B}$, let $f()=.O R(\mathrm{R})$, and assume that $\mathrm{A}=\mathrm{S}_{1} \times \ldots \times \mathrm{S}_{\mathrm{i}}$ $\times \ldots \times \mathrm{S}_{\mathrm{j}} \times \ldots \times \mathrm{S}_{\mathrm{n}} \times \mathrm{I}$ and $\mathrm{B}=\mathrm{S}_{1} \times \ldots \times \mathrm{S}_{\mathrm{i}} \times \ldots \times \mathrm{S}_{\mathrm{j}} \times \ldots \times \mathrm{S}_{\mathrm{n}} \times \mathrm{O}$. Let $f(\ldots)$ be a function that implements R. Let $f_{l}(\ldots)$ and $f_{2}(\ldots)$ be two functions with domains $\mathrm{S}_{1} \times \ldots \times \mathrm{S}_{\mathrm{i}}$ $\times \ldots \times \mathrm{S}_{\mathrm{j}} \times \ldots \times \mathrm{S}_{\mathrm{n}} \times \mathrm{I}$. If $f\left(x_{1}, \ldots, x_{i}, x_{i+1}, \ldots, x_{n}, i\right)=\left\langle x_{1}{ }_{1}, \ldots, x_{i}{ }_{i}, x^{\prime}{ }_{i+1}, \ldots, x_{n}, o\right\rangle$, we use the notation $f_{i i}$ to refer to the projection of $f$ over the set $\mathrm{S}_{\mathrm{i}}$, i.e., $f_{1 i}\left(x_{1}, \ldots, x_{i}, x_{i+1}, \ldots, x_{n}, i\right)=$ $x^{\prime}{ }_{i}$. Similarly, we define $f_{\mathrm{I} S}$ as the projection of $f$ over the set $\mathrm{S}=\mathrm{S}_{\mathrm{i}} \times \ldots \times \mathrm{S}_{\mathrm{j}}$ for some $i$ and $j$. Let $\operatorname{Ref}(f)$ be the set of variables used in the computation of $f(\ldots)$ and $\operatorname{Mod}(f)$ be the set of variables modified by $f(\ldots)$ be the set of state variables that are modified by $f$, i.e. the set of variables $\left\{x_{i}\right\}_{\mathrm{i}}$ such that $f_{\mathrm{li}}\left(x_{1}, \ldots, x_{i}, x_{i+1}, \ldots, x_{n}, i\right)=x_{i}{ }_{i} \neq x_{i}$. A function $f(\ldots)$ is said to be effectively sliceable if and only if there exist two functions $f_{l}\left(x_{1}, \ldots\right.$ $\left.x_{n}, i\right)$ and $f_{2}\left(x_{1}, \ldots x_{n}, i\right)$ such that:

$$
\begin{aligned}
\operatorname{Mod}\left(f_{1}\right) \cap \operatorname{Ref}\left(f_{2}\right)= & \Phi, \operatorname{Mod}\left(f_{2}\right) \cap \operatorname{Ref}\left(f_{1}\right)=\Phi, \operatorname{Mod}\left(f_{1}\right) \cap \operatorname{Mod}\left(f_{2}\right)=\Phi \\
& \operatorname{Mod}\left(f_{1}\right) \cup \operatorname{Mod}\left(f_{2}\right)=\operatorname{Mod}(f),
\end{aligned}
$$




$$
\begin{gathered}
f\left|\bmod (f)\left(x_{1}, \ldots, x_{n}, i\right)=f_{1 \mid \operatorname{Mod}(\mathrm{f} 1)}\left(x_{1}, \ldots, x_{n}, i\right) \bullet f_{2}\right| \operatorname{Mod}(\mathrm{f} 2)\left(x_{1}, \ldots, x_{n}, i\right), \text { and } \\
f_{\mid o}\left(x_{1}, \ldots, x_{n}, i\right)=f_{1 \mid o}\left(x_{1}, \ldots, x_{n}, i\right) \bullet f_{2} \mid o\left(x_{1}, \ldots, x_{n}, i\right)
\end{gathered}
$$

for some ordering of the state variables $x_{1}, \ldots, x_{n}$. Figure 3 illustrates the first three equalities in a Venn Diagram. Note that a sliceable function is also effectively sliceable. An interesting property of effectively sliceable functions is that the component functions may be executed in any sequence. There are other cases of sliceability, but in this case, the subfunctions have to be executed in a particular order. We call this temporal sliceability. Temporal sliceability is a weaker condition than effective sliceability, and is described as follows. Let $\mathrm{R} \subseteq \mathrm{A} \times \mathrm{B}$, let $f()=.O R(\mathrm{R})$, and assume that $\mathrm{A}$ $=\mathrm{S}_{1} \times \ldots \times \mathrm{S}_{\mathrm{i}} \times \ldots \times \mathrm{S}_{\mathrm{j}} \times \ldots \times \mathrm{S}_{\mathrm{n}} \times \mathrm{I}$ and $\mathrm{B}=\mathrm{S}_{1} \times \ldots \times \mathrm{S}_{\mathrm{i}} \times \ldots \times \mathrm{S}_{\mathrm{j}} \times \ldots \times \mathrm{S}_{\mathrm{n}} \times \mathrm{O}$. Let $f(\ldots)$ be a function that implements R. Let $f_{l}(\ldots)$ and $f_{2}(\ldots)$ be two functions with domains $\mathrm{S}_{1} \times \ldots \times \mathrm{S}_{\mathrm{i}} \times \ldots \times \mathrm{S}_{\mathrm{j}} \times \ldots \times \mathrm{S}_{\mathrm{n}} \times \mathrm{I}$. Using the same notation as above, we say that function $f(\ldots)$ is said to be temporally sliceable if and only if there exist two functions $f_{1}\left(x_{1}, \ldots\right.$ $\left.x_{n}, i\right)$ and $f_{2}\left(x_{1}, \ldots x_{n}, i\right)$ such that:

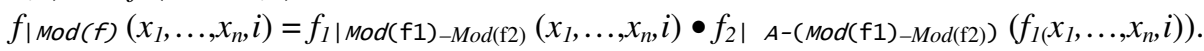

$\operatorname{Mod}\left(f_{1}\right)-\operatorname{Mod}\left(f_{2}\right)$ represents the set of variables that are modified by $f_{1}$ but not by $f_{2}$. Some of these variables may, however, be referenced by $\mathrm{f} 2$ and we don't care about that. Obviously, the relationship between $f_{1}$ and $f_{2}$ is not a symmetrical one, and the functions have to be executed in a particular order.

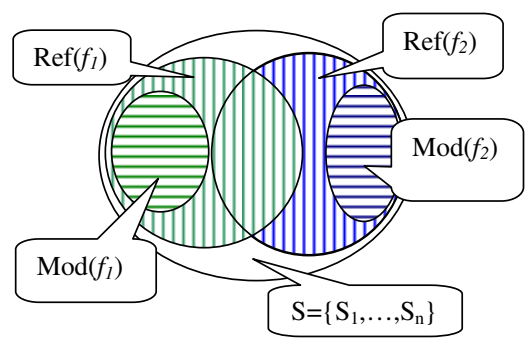

Fig. 3. A function is effectively sliceable if it can be written as the concatenation of two functions that modify disjoint parts of an object, and don't refer to the parts that the others modify

In [11], we showed that provided that methods of objects do not modify objects other than the executing ones, any method that computes a function and modifies the receiver object can be decomposed into a sequence of pure functional and purely sideeffectal functions. To compose two hybrid functions, we decompose them along the purely functional versus purely side-effectal dimensions, find the smallest granularity decomposition between the two, and then compose them slice-by-slice.

The major problem, of course, is our tendency to code "service-oriented functions", i.e. functions that are application level but that are coded at the domain class level. These functions are not composable because they address an application specific need, each. You would want to compose them because they embody a general behavior that is not encapsulated elsewhere. Obviously, not choosing the right granularity is a problem, and leads to methods that are not composable. 


\section{Discussion}

This is a very preliminary investigation into the principles of separation of concerns and the foundations of the techniques that promote separation of concerns. The yardstick by which innovations in software engineering are to be assessed has always been-and rightly so- to determine the problem that a given method, technique, or tool, solves. Separation of concerns is only useful to the extent that once the concerns have been addressed separately, we are able to re-combine the individual and partial solutions into one that addresses all of them.

Some of the case studies that are available in the literature show cases where concern separation is difficult in practice [7], [8], [9]. Others showed that aspect/subject composition is difficult, even in cases where the aspects or subjects embody distinctly different concerns [9], [11], [15]. We attempted to frame the separation of concerns in software development in terms of homomorphisms of development transformations, and then we tried to determine the "operating range" of these homomorphisms. This preliminary work raised more questions than it answered, and some of the answers are reassuringly common-sensical, but are worth stating:

- Not all requirements (concerns) are composable in the sense that they lead to composable artifacts. Viewing requirements as input-output relations, we identified simple conditions on the domains and images of these requirements, which essentially say that the requirements should not be conflicting. In particular, method cancellation through subject composition or aspect weaving is no less dangerous than cancellation with inheritance: they are both a sign of either a violation of intent, or of sloppy realization (implementation).

- We should treat aspects that embody run-time requirements differently-and separately-from aspects that embody functional (domain) requirements. We framed run-time requirements (persistence, fault-tolerance, etc.) in terms of functional requirements of the virtual machine. In an ideal world, such concerns should also be handled by virtual machine - or more generally, meta-level-aspects, and a number of recent approaches have gone that route. However, other considerations, such as performance, security, integrity, or portability, may suggest otherwise at the risk of inducing composability problems.

- Not all programs that implement several concerns can be reengineered into separate aspects. The underlying concerns/requirements may not be separable (essential inseparability), or the current implementation may not lend itself to such a separation (accidental inseparability). Object slicing helps with accidental inseparability.

We have started to take a closer look at the existing AOSD methods and the case studies to judge the usefulness of the above framework. We were able to explain known difficulties with subject-oriented composition (see e.g. [14]) and view attachment [13] in terms of violations of some of the principles outlined above. We have also started looking at the broken delegation problem from the perspective of functional composability and separability. The broken delegation problem manifests itself when we use aggregation (and message forwarding) as a way of compose behaviors. The problem is often referred to as an all-or-nothing problem. The "self" in an object component is either used to refer to the component-in which case we have broken delegation - or to the entire object, in which case, we do not have a problem. We have 
shown elsewhere that attempts to fix the broken delegation problem can seriously compromise application security, and what we need is a more analytical approach to the problem. Our approach enables us to frame the problem.

\section{References}

1. D. Bardou \& C. Dony, "Split objects: a disciplined use of delegation within objects," in proc. OOPSLA'96, 1996.

2. L. Bass, P. Clements \& R. Kazman, Software Architecture in Practice, 1998.

3. I. Baxter, "Design Maintenance Systems," CACM, vol. 35, no. 4, April 1992, pp. 73-89.

4. S. Dasgupta, "The Nature of Design Problems," in Design Theory and Computer Science, Cambridge University Press, 1991, pp. 13-35.

5. R. E. Filman \& D. P. Friedman, "Aspect-oriented programming is quantification and obliviousness," in proc. of OOPSLA workshop on Advanced Separation of Concerns, 2000.

6. W. Harrison \& H.Ossher, "Subject-oriented programming: a critique of pure objects," in Proc. OOPSLA'93, 1993.

7. S. Herrmann \& M. Mezini, "On the Need for a Unified MDSOC Model: Experiences from Constructing a Modular Software Engineering Environment", MSDOC workshop, OOPSLA'00, 2000

8. E. A. Kendall. Role Model Designs and Implementations with Aspect Oriented Programming. In Proc. OOPSLA'99, 1999

9. M. Kersten \& G. Murphy, "Atlas: a case study in building a Web-based learning environment using aspect-oriented programming", in proc. OOPSLA'99, 1999.

10. G. Kiczales, J. Lamping, C. Lopez, “Aspect-Oriented Programming,” in Proc. ECOOP’97.

11. H. Mili, "On behavioral descriptions in oject-oriented modeling", Journal of Systems and Software, 1996.

12. H. Mili, A. Mili, J. Dargham, O. Cherkaoui \& R. Godin, "View Programming: Towards a Framework for Decentralized Development and Execution of OO Programs," Proceedings of TOOLS USA '99, 1999.

13. H. Mili, H. Mcheick \& J. Dargham, "CorbaViews: Distribting objects with several functional aspects," Journal of Object Technology, August 2002,.

14. H. Ossher, M. Kaplan, W. Harrison, A. Katz, and V. Kruskal, "Specifying subject-oriented composition," in TAPOS, 2(3), 1996.

15. M. Robillard \& G. Murphy, "Analyzing Concerns Using Class Member Dependencies," Position paper for the ICSE Workshop on Advanced Separation of Concerns in SE, 2001.

16. H. A. Simon, Models of bounded rationality, vol. 2, Cambridge, MA (MIT Press, 1982).

17. T. Soued, N. Yahiaoui, L. Seinturier, B. Traverson, "Techniques d'aspect pour la gestion de la mémoire répartie dans un environnement CORBA/C++", in proc. of NOTERE'05, 2005.

18. T. Reenskaugh, in Working with Objects, Prentice-Hall, 1995.

19. F. Steimann, "Domain Models are Aspect Free," in Proc of MODELS'05, 2005.

20. F. Tip, J-D Choi, J. Field, and G. Ramalingam, "Slicing class hierarchies in C++", In Proc. of OOPSLA'96, 1966.

21. P. Zave and M. Jackson, "Conjunction as Composition," in ACM Trans. on Softw. Eng. Methodol., vol. 2, no. 4, pp. 379-411, 1993. 